\title{
QATAR-2: A K DWARF ORBITED BY A TRANSITING HOT JUPITER AND A MORE MASSIVE COMPANION IN AN OUTER ORBIT
}

\author{
Marta L. Bryan ${ }^{1}$, Khalid A. Alsubai ${ }^{2}$, David W. Latham ${ }^{3}$, Neil R. Parley ${ }^{4}$, Andrew Collier Cameron ${ }^{4}$, \\ Samuel N. Quinn ${ }^{3}$, Joshua A. Carter ${ }^{3,12}$, Benjamin J. Fulton ${ }^{5}$, Perry Berlind ${ }^{3}$, Warren R. Brown ${ }^{3}$, \\ Lars A. Buchhave ${ }^{6,7}$, Michael L. Calkins ${ }^{3}$, Gilbert A. Esquerdo ${ }^{3}$, Gábor Fúrész ${ }^{3,13}$, Uffe Gråe Jørgensen ${ }^{6,7}$, \\ Keith D. Horne ${ }^{4}$, Robert P. Stefanik ${ }^{3}$, Rachel A. Street ${ }^{5}$, Guillermo Torres ${ }^{3}$, Richard G. West ${ }^{8}$, Martin Dominik ${ }^{4,14}$, \\ Kennet B. W. Harps $ø E^{6,7}$, Christine Liebig ${ }^{4}$, Sebastiano Calchi Novati ${ }^{9}, 10$, Davide Ricci ${ }^{11}$, and Jesper F. Skottfelit ${ }^{6,7}$ \\ ${ }^{1}$ Department of Astronomy, Harvard University, Cambridge, MA 02138, USA \\ ${ }^{2}$ Qatar Foundation, P.O. Box 5825, Doha, Qatar \\ ${ }^{3}$ Harvard-Smithsonian Center for Astrophysics, 60 Garden Street, Cambridge, MA 02138, USA \\ ${ }^{4}$ SUPA, School of Physics and Astronomy, University of St Andrews, North Haugh, St Andrews, Fife KY16 9SS, UK \\ ${ }^{5}$ Las Cumbres Observatory Global Telescope Network, 6740 Cortona Drive, Suite 102, Goleta, CA 93117, USA \\ ${ }^{6}$ Niels Bohr Institute, University of Copenhagen, Juliane Maries vej 30, 2100 Copenhagen, Denmark \\ ${ }^{7}$ Centre for Star and Planet Formation, Geological Museum, Øster Voldgade 5, 1350 Copenhagen, Denmark \\ ${ }^{8}$ Department of Physics and Astronomy, University of Leicester, Leicester LE1 7RH, UK \\ ${ }^{9}$ Dipartimento di Fisica "E. R. Caianiello," Università degli Studi di Salerno, Via S. Allende, 84081 Baronissi (SA), Italy \\ ${ }^{10}$ INFN, Gruppo Collegato di Salerno, Sezione di Napoli, Italy \\ ${ }^{11}$ Institut d'Astrophysique et de Géophysique, Allée du 6 Août 17, Sart Tilman, Bât. B5c, 4000 Liège, Belgium \\ Received 2011 October 21; accepted 2012 February 19; published 2012 April 17
}

\begin{abstract}
We report the discovery and initial characterization of Qatar-2b, a hot Jupiter transiting a $V=13.3$ mag K dwarf in a circular orbit with a short period, $P_{\mathrm{b}}=1.34$ days. The mass and radius of Qatar- $2 \mathrm{~b}$ are $M_{\mathrm{P}}=2.49 M_{\mathrm{J}}$ and $R_{\mathrm{P}}=1.14 R_{\mathrm{J}}$, respectively. Radial-velocity monitoring of Qatar-2 over a span of 153 days revealed the presence of a second companion in an outer orbit. The Systemic Console yielded plausible orbits for the outer companion, with periods on the order of a year and a companion mass of at least several $M_{\mathrm{J}}$. Thus, Qatar-2 joins the short but growing list of systems with a transiting hot Jupiter and an outer companion with a much longer period. This system architecture is in sharp contrast to that found by Kepler for multi-transiting systems, which are dominated by objects smaller than Neptune, usually with tightly spaced orbits that must be nearly coplanar.
\end{abstract}

Key words: planetary systems - stars: individual (Qatar-2) - techniques: photometric - techniques: radial velocities - techniques: spectroscopic

Online-only material: color figures, supplemental data (FITS) file (tar.gz)

\section{INTRODUCTION}

Wide-angle ground-based photometric surveys, such as WASP and HATNet, have been effective for the identification of close-in exoplanets that transit their host stars. Although most of the systems found by these surveys are fainter than the stars targeted by Doppler surveys, they are still bright enough to allow confirmation and characterization of their transiting planets using follow-up photometric and spectroscopic observations.

Most of the more than 150 confirmed transiting planets are hot Jupiters. Of those found by ground-based surveys, only 12 are smaller than Saturn, i.e., less than 9.4 Earth radii (Schneider 2011; as of 2011 September). In contrast, most of the more than 1000 candidates identified by Kepler are smaller than Neptune, i.e., less than 3.8 Earth radii (e.g., see Latham et al. 2011). However, it will not be possible to determine spectroscopic orbits for the vast majority of Kepler's small candidates because the required velocity precision is beyond the reach of present capabilities with instruments such as the High Resolution Echelle Spectrometer on Keck I (e.g., see Batalha et al. 2011).

Part of the problem is that most of the Kepler candidates are 14th magnitude or fainter, a result of the fact that Kepler

\footnotetext{
${ }^{12}$ Hubble Fellow.

${ }^{13}$ Also at Konkoly Observatory of the Hungarian Academy of Sciences, Budapest, Hungary.

${ }^{14}$ Royal Society University Research Fellow.
}

monitors a region that is only $0.25 \%$ of the sky. To find all of the nearest and brightest transiting planets, we now need to extend our photometric surveys to cover the entire sky. The prospects are good that such surveys will eventually be pursued from space, with missions such as TESS (an Explorer selected for Phase A by NASA) and PLATO (proposed to ESA), but the earliest any of those spacecraft could be launched is 2016 .

In the meantime, at least three teams are working on groundbased photometric surveys designed to find the best systems for follow-up studies. An obvious strategy is to cover as much of the sky as possible (ideally all), so that no prime systems are missed. A complementary strategy is to target smaller stars, where smaller planets are easier to detect and characterize. The MEarth project has adopted both strategies by focusing on the coolest and smallest $\mathrm{M}$ dwarfs, targeting a few thousand of the nearest and brightest examples all over the sky. This approach has already yielded GJ 1214b (Charbonneau et al. 2009), which lies in the "Super-Earth" transition region between Neptune and the Earth and has attracted a lot of interest because its favorable contrast with its host star allows studies of its atmosphere (e.g., see Bean et al. 2011). The other two efforts, the extensions of HATNet to HAT South and of WASP to the Qatar Exoplanet Survey (QES), have chosen to improve the capability of the more traditional strategy of a magnitudelimited wide-angle survey. Both HAT South and QES have implemented cameras with larger apertures, thus providing 
better photometric precision and lower rates of contamination by faint stars close to the targets (enabled by the more favorable pixel scale resulting from the longer focal lengths of the larger cameras). An important trade-off of this approach is that it requires more detector pixels to cover the same area on the sky.

A practical implication of finding smaller planets is that the follow-up Doppler spectroscopy needed to determine orbits and planetary masses will demand access to the most capable facilities for very precise radial velocities, such as HARPS on the European Southern Observatory's 3.6 m telescope on la Silla in the Southern Hemisphere, and HARPS-N, scheduled to come into operation in 2012 on the Telescopio Nazionale Galileo $3.6 \mathrm{~m}$ telescope operated for the Italian Institute of Astrophysics on La Palma in the Northern Hemisphere.

In this paper we report the discovery and initial characterization of Qatar-2b, the second transiting planet from QES to be confirmed (for the first, see Alsubai et al. 2011). Qatar-2b is especially interesting because our radial-velocity monitoring shows that there is a second companion in the system with an orbital period of about a year and a mass of at least several $M_{\mathrm{J}}$.

\section{QES DISCOVERY PHOTOMETRY}

QES is a wide-field photometric survey for transiting planets. The initial five-camera CCD imaging system is now deployed at a site in New Mexico and has been in operation for more than two years. It uses an array of five Canon lenses equipped with $4 \mathrm{~K} \times 4 \mathrm{~K} \mathrm{CCDs}$ to image an $11^{\circ} \times 11^{\circ}$ field on the sky simultaneously at two different pixel scales (Alsubai et al. 2011). The data are reduced at the University of St. Andrews using pipeline software based on the image-subtraction algorithm of Bramich (2008), and the data products are archived at the University of Leicester, using the same architecture as the WASP archive (Pollacco et al. 2006).

The QES photometry for the $V=13.3$ mag K dwarf that we now designate Qatar-2 (3UC 167-129863, $\alpha_{2000}=13^{\mathrm{h}} 50^{\mathrm{m}} 37^{\mathrm{s}} .41$, $\delta_{2000}=-06^{\circ} 48^{\prime} 14^{\prime \prime}$. ; Zacharias et al. 2010) revealed transit-like events, found by an automated search on the archive data using the box least-squares algorithm of Kovács et al. (2002) as modified for the SuperWASP project by Collier Cameron et al. (2006). Systematic patterns of correlated noise were modeled and removed from the archive light curves using a combination of the SysRem algorithm of Tamuz et al. (2005) and the trend filtering algorithm of Kovács et al. (2005). 3UC 167-129863 was screened and selected as a promising candidate, along with two other stars in the same QES field, using tests described by Collier Cameron et al. (2007), designed to ensure that the depths and durations of observed transits are consistent with the expectations for objects of planetary dimensions transiting main-sequence stars. The discovery light curve, shown in Figure 1, indicated an ephemeris of $E=2455618.9092 \pm 0.0014+N \times 1.337098 \pm 0.000022$ BJD.

\section{FOLLOW-UP SPECTROSCOPY WITH TRES}

We monitored the spectrum of Qatar-2 using the Tillinghast Reflector Echelle Spectrograph (TRES), mounted on the $1.5 \mathrm{~m}$ Tillinghast Reflector at the Fred L. Whipple Observatory on Mount Hopkins, Arizona. Over a period of 153 days from 2011 January 18 to June 21 we obtained 44 spectra with a signalto-noise ratio per resolution element (SNRe) of at least 20 in the continuum at the center of the order containing the $\mathrm{Mg} \mathrm{b}$ features (near $518 \mathrm{~nm}$ ). We used the medium fiber, which yields

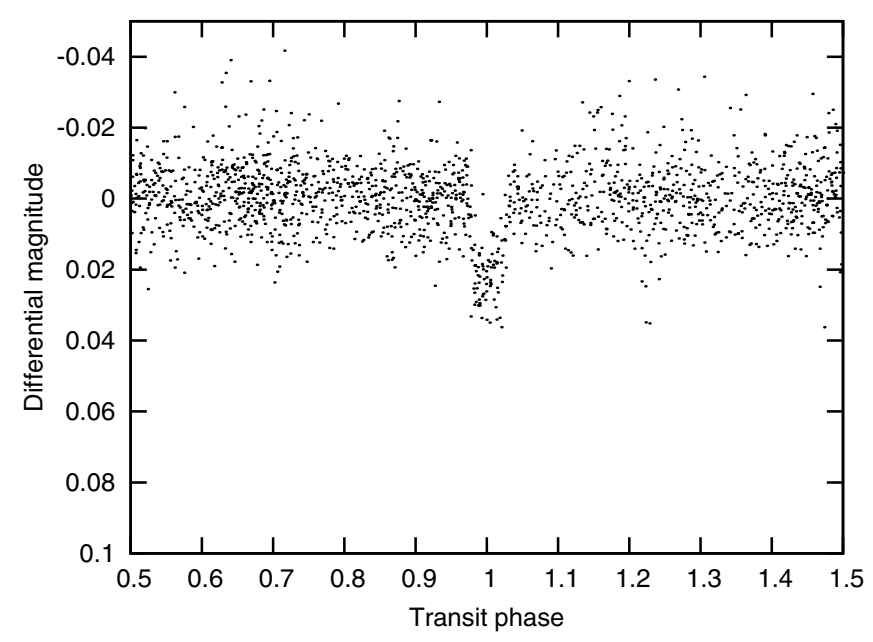

Figure 1. QES discovery light curve, phased on the transit ephemeris of Qatar-2b. The data shown here were obtained with Camera 403 in campaigns $\mathrm{C} 2, \mathrm{C} 3$, and $\mathrm{C} 4$.

a resolving power of $R \sim 44,000$, corresponding to a resolution element with $6.8 \mathrm{~km} \mathrm{~s}^{-1}$ FWHM. The spectra were extracted using the procedures outlined by Buchhave et al. (2010). The wavelength calibration was established using exposures of a thorium-argon hollow-cathode lamp illuminating the fiber, immediately before and after the stellar observations. A typical exposure time for a stellar observation was 45 minutes.

\subsection{Radial Velocities}

We derived radial velocities from the TRES spectra using the procedures for multi-order cross correlations described by Buchhave et al. (2010). We present here a brief summary of the technique. First, we rejected roughly half of the echelle orders: several orders with low signal-to-noise ratio shortward of $446 \mathrm{~nm}$; orders longward of $678 \mathrm{~nm}$, which are contaminated by telluric absorption lines or show strong fringing in the CCD; and a few orders in between which are known to give poor velocity performance relative to the other orders (generally because of some other contamination such as interstellar $\mathrm{Na}$ D absorption or emission lines from Earth's atmosphere). In all, we included 23 orders in our analysis. Each observed spectrum was cross correlated, order by order, against the corresponding orders from the strongest single observation, the one obtained on JD 2455646. The cross correlation functions (CCFs) from the individual orders were summed and fit with a Gaussian function to determine the radial velocity. We estimated the internal precision of the radial velocity for each observation by fitting a Gaussian to the CCFs of the individual orders and using the deviations from the global fit to calculate the standard deviation of the mean.

We adjusted the velocities to correct for small shifts in the zero point between observing runs, which mostly resulted from minor modifications to the hardware between some runs. These modifications included installing a new front end and fiber feed in 2011 February, which shifted the velocity zero point by $60 \mathrm{~m} \mathrm{~s}^{-1}$ for the run starting on BJD 2455602. An adjustment to the focus of TRES after the end of that run brought the shift back by $26 \mathrm{~m} \mathrm{~s}^{-1}$. We observed three IAU radial-velocity standard stars every night, and were able to establish the run-torun shifts with an uncertainty on the order of $5 \mathrm{~m} \mathrm{~s}^{-1}$. The three observed standard stars, HD 3765, 38230, and 182488, have all been demonstrated to be constant to a level of 1 or $2 \mathrm{~m} \mathrm{~s}^{-1} \mathrm{yr}^{-1}$ 
Table 1

Relative Radial Velocities of Qatar-2

\begin{tabular}{|c|c|c|c|c|c|}
\hline BJD & $\begin{array}{c}\mathrm{RV}^{\mathrm{a}} \\
\left(\mathrm{m} \mathrm{s}^{-1}\right)\end{array}$ & $\begin{array}{c}\sigma_{\mathrm{RV}}^{\mathrm{b}} \\
\left(\mathrm{m} \mathrm{s}^{-1}\right)\end{array}$ & $\begin{array}{c}\mathrm{BS}^{\mathrm{c}} \\
\left(\mathrm{m} \mathrm{s}^{-1}\right)\end{array}$ & $\begin{array}{c}\sigma_{\mathrm{BS}} \\
\left(\mathrm{m} \mathrm{s}^{-1}\right)\end{array}$ & $\begin{array}{l}\text { Zero-point Corrections } \\
\qquad\left(\mathrm{m} \mathrm{s}^{-1}\right)\end{array}$ \\
\hline 2455580.011622 & 231.4 & 33.8 & 18.8 & 23.2 & -33.5 \\
\hline 2455581.027117 & 383.2 & 37.7 & -31.5 & 22.6 & -33.5 \\
\hline 2455583.034587 & -581.6 & 26.7 & -3.8 & 16.8 & -33.5 \\
\hline 2455583.961503 & 276.4 & 32.6 & -9.7 & 17.1 & -33.5 \\
\hline 2455585.003598 & 327.3 & 27.2 & -6.7 & 13.8 & -33.5 \\
\hline 2455587.983438 & 271.2 & 27.4 & -15.0 & 18.6 & -33.5 \\
\hline 2455602.958910 & -396.5 & 32.9 & 12.2 & 16.0 & 26.0 \\
\hline 2455604.034894 & 210.7 & 29.6 & 2.7 & 21.0 & 26.0 \\
\hline 2455604.966229 & 59.3 & 29.2 & 5.3 & 25.4 & 26.0 \\
\hline 2455605.966418 & -737.8 & 23.6 & 23.7 & 12.3 & 26.0 \\
\hline 2455607.001348 & -536.2 & 32.9 & -22.0 & 18.0 & 26.0 \\
\hline 2455607.987854 & 346.7 & 35.5 & 15.8 & 25.2 & 26.0 \\
\hline 2455608.955271 & -93.6 & 27.7 & -21.6 & 14.9 & 26.0 \\
\hline 2455610.996605 & -447.0 & 36.5 & 4.2 & 25.2 & 26.0 \\
\hline 2455615.938163 & 285.1 & 23.9 & 3.8 & 15.8 & -3.1 \\
\hline 2455616.973605 & -71.0 & 28.9 & -10.2 & 23.1 & -3.1 \\
\hline 2455617.978176 & -799.2 & 21.0 & -30.7 & 13.6 & -3.1 \\
\hline 2455643.881697 & 214.0 & 29.1 & 19.7 & 17.1 & 0.0 \\
\hline 2455644.849974 & -638.7 & 25.5 & 14.6 & 12.6 & 0.0 \\
\hline 2455645.901592 & -802.6 & 20.6 & -4.8 & 20.6 & 0.0 \\
\hline 2455646.846694 & 0.0 & 17.4 & -28.8 & 11.6 & 0.0 \\
\hline 2455647.890953 & 160.3 & 35.4 & -16.8 & 19.1 & 0.0 \\
\hline 2455650.857343 & 57.0 & 27.1 & 2.1 & 15.3 & 0.0 \\
\hline 2455652.895515 & -603.6 & 32.9 & -9.2 & 19.4 & 0.0 \\
\hline 2455656.870962 & -711.0 & 22.2 & -25.0 & 10.9 & 3.2 \\
\hline 2455659.877783 & 119.4 & 25.4 & -0.8 & 17.5 & 3.2 \\
\hline 2455662.839965 & 171.5 & 29.1 & -0.4 & 21.5 & 3.2 \\
\hline 2455663.812865 & -83.3 & 21.3 & -7.8 & 15.6 & 3.2 \\
\hline 2455664.867650 & -764.9 & 30.3 & 5.6 & 21.7 & 3.2 \\
\hline 2455665.796571 & -549.1 & 21.3 & 2.3 & 14.6 & 3.2 \\
\hline 2455668.845188 & -835.7 & 30.1 & 158.4 & 48.5 & 3.2 \\
\hline 2455671.762510 & -211.4 & 21.8 & 111.4 & 36.9 & 3.2 \\
\hline 2455672.733966 & -850.1 & 28.6 & 26.5 & 13.1 & 3.2 \\
\hline 2455673.784772 & -471.2 & 19.2 & -16.2 & 14.9 & 3.2 \\
\hline 2455691.770974 & -292.0 & 32.0 & 13.4 & 20.3 & 0.4 \\
\hline 2455702.699240 & 229.8 & 24.2 & -22.9 & 19.0 & 0.4 \\
\hline 2455703.727260 & -471.1 & 32.2 & -22.6 & 17.6 & 0.4 \\
\hline 2455704.730193 & -724.7 & 19.5 & -20.6 & 12.5 & 0.4 \\
\hline 2455705.694921 & 148.7 & 42.0 & -13.7 & 28.5 & 0.4 \\
\hline 2455706.738098 & 258.4 & 27.6 & -15.8 & 15.8 & 0.4 \\
\hline 2455722.744329 & 334.0 & 33.7 & 4.6 & 25.0 & -2.1 \\
\hline 2455726.718494 & 269.1 & 30.1 & -16.9 & 23.6 & -2.1 \\
\hline 2455728.755461 & -500.9 & 31.5 & -10.7 & 22.1 & -2.1 \\
\hline 2455733.706680 & 407.0 & 27.5 & -60.7 & 28.9 & -2.1 \\
\hline
\end{tabular}

Notes.

a The zero point of these velocities is relative to the observation obtained on BJD 2455646. The absolute velocity of that observation on the IAU system is $-23.8 \pm 0.1 \mathrm{~km} \mathrm{~s}^{-1}$.

b Internal errors, summed in quadrature with the uncertainty of the run-to-run zero-point shifts, assumed to be $5 \mathrm{~m} \mathrm{~s}^{-1}$.

$\mathrm{c}$ The zero point of the bisector spans is arbitrary.

(HD 182488 has a slow drift due to a distant companion). These adjustments have been applied to the individual velocities for Qatar-2 reported in Table 1, which are relative to the observation obtained on JD 2455646. The absolute velocity of that observed template was derived using our observations of IAU radialvelocity standard stars and correlations of the $\mathrm{Mg} b$ order against a library of synthetic templates, yielding a value of $-23.8 \pm 0.1 \mathrm{~km} \mathrm{~s}^{-1}$ on a system where the velocity of our primary standard, HD 182488 , is defined to be $-21.508 \mathrm{~km} \mathrm{~s}^{-1}$. The error estimate for this absolute velocity is fairly large,
Table 2

Key Orbital Parameters

\begin{tabular}{lcc}
\hline \hline Parameter & Quadratic & Systemic $^{\mathrm{a}}$ \\
\hline$P_{\mathrm{b}}($ days $)$ & $1.337169 \pm 0.000076$ & $1.337148 \pm 0.000095$ \\
$K_{\mathrm{b}}\left(\mathrm{m} \mathrm{s}^{-1}\right)$ & $559 \pm 6$ & $559 \pm 6$ \\
$M_{\mathrm{b}}\left(M_{\mathrm{J}}\right)$ & $2.48 \pm 0.03$ & $2.49 \pm 0.05$ \\
$e_{\mathrm{b}}$ & $0.01 \pm 0.01$ & $0.003 \pm 0.008$ \\
$P_{\mathrm{c}}($ days $)$ & $\ldots$ & $332::$ \\
$K_{\mathrm{c}}\left(\mathrm{m} \mathrm{s}^{-1}\right)$ & $\ldots$ & $301::$ \\
$M_{\mathrm{c}}\left(M_{\mathrm{J}}\right)$ & $\ldots$ & $8.4::$ \\
$e_{\mathrm{c}}$ & $\ldots$ & $0.09::$ \\
$\Delta \gamma^{\mathrm{b}}\left(\mathrm{m} \mathrm{s}^{-1}\right)$ & $-282.6 \pm 5.2$ & $-65::$ \\
$d \gamma / d t\left(\mathrm{~m} \mathrm{~s}^{-1}\right.$ day $\left.^{-1}\right)$ & $-2.76 \pm 0.16$ & $\ldots$ \\
$d^{2} \gamma / d t^{2}\left(\mathrm{~m} \mathrm{~s}^{-1}\right.$ day $\left.^{-2}\right)$ & $0.0875 \pm 0.0046$ & $\cdots$ \\
\hline
\end{tabular}

Notes.

a This is a typical solution from the Systemic Console; we do not quote errors for the parameters of the outer orbit because the Systemic Console yielded several plausible solutions, with differences much larger than the internal error estimates. The parameters given here for the outer companion should be treated with extreme caution.

${ }^{\mathrm{b}} \Delta \gamma$ is the offset of the center-of-mass velocity for the set of relative velocities used for the orbital solution and reported in Table 1.

because only a single echelle order was used in the comparison with HD 182488 by way of the synthetic library spectra, and the absolute velocity of HD 182488 itself has an uncertainty at the level of nearly $0.1 \mathrm{~km} \mathrm{~s}^{-1}$. Of course, our orbital solutions depend only on the relative velocities, which are much more precise.

\subsection{Orbital Solution}

We ran a Markov Chain Monte Carlo (MCMC) analysis on the radial-velocity data, starting with Gaussian constraints on the priors for period and epoch from the QES discovery photometry. We found that a single Keplerian orbit matched the data poorly, with $\chi^{2}=424.9$ for 44 observations and 38 degrees of freedom. Introducing two additional terms, for a linear and quadratic velocity drift in a Taylor expansion, resulted in a dramatic improvement to the fit, with $\chi^{2}=46.0$ for 36 degrees of freedom (reduced $\chi^{2}=1.28$ ). This model provided convincing evidence that Qatar-2 is orbited by a transiting hot Jupiter, Qatar-2b, with period $P=1.34$ days, plus a second companion, Qatar-2c, with a period of roughly a year. The velocity curves for the orbit of Qatar-2b (phased to the period) and the acceleration due to Qatar-2c are plotted in the bottom two sections of Figure 2, together with the corresponding components of the observed velocities. The top panel shows the original velocities together with the combined velocity model. The panels immediately under the velocity curves show the residuals from the fits.

Next, we used the Systemic Console (Meschiari et al. 2009) to explore possible simultaneous fits for two Keplerian orbits. After taking the mass of Qatar-2 to be $0.782 M_{\odot}$, a Levenberg-Marquardt solution gave a plausible fit and provided starting parameters for an MCMC analysis. When the eccentricities were allowed to be free parameters, several plausible solutions with similar parameters were found, all with periods a little shorter than a year and companion masses of several $M_{\mathrm{J}}$. The key parameters for a typical solution from the Systemic Console are reported in Table 2, along with the parameters from the quadratic solution. Since the eccentricities for both the inner and outer planet were not significantly different from circular at $0.003 \pm 0.008$ and $0.09 \pm 0.07$, respectively, we did not quote the 

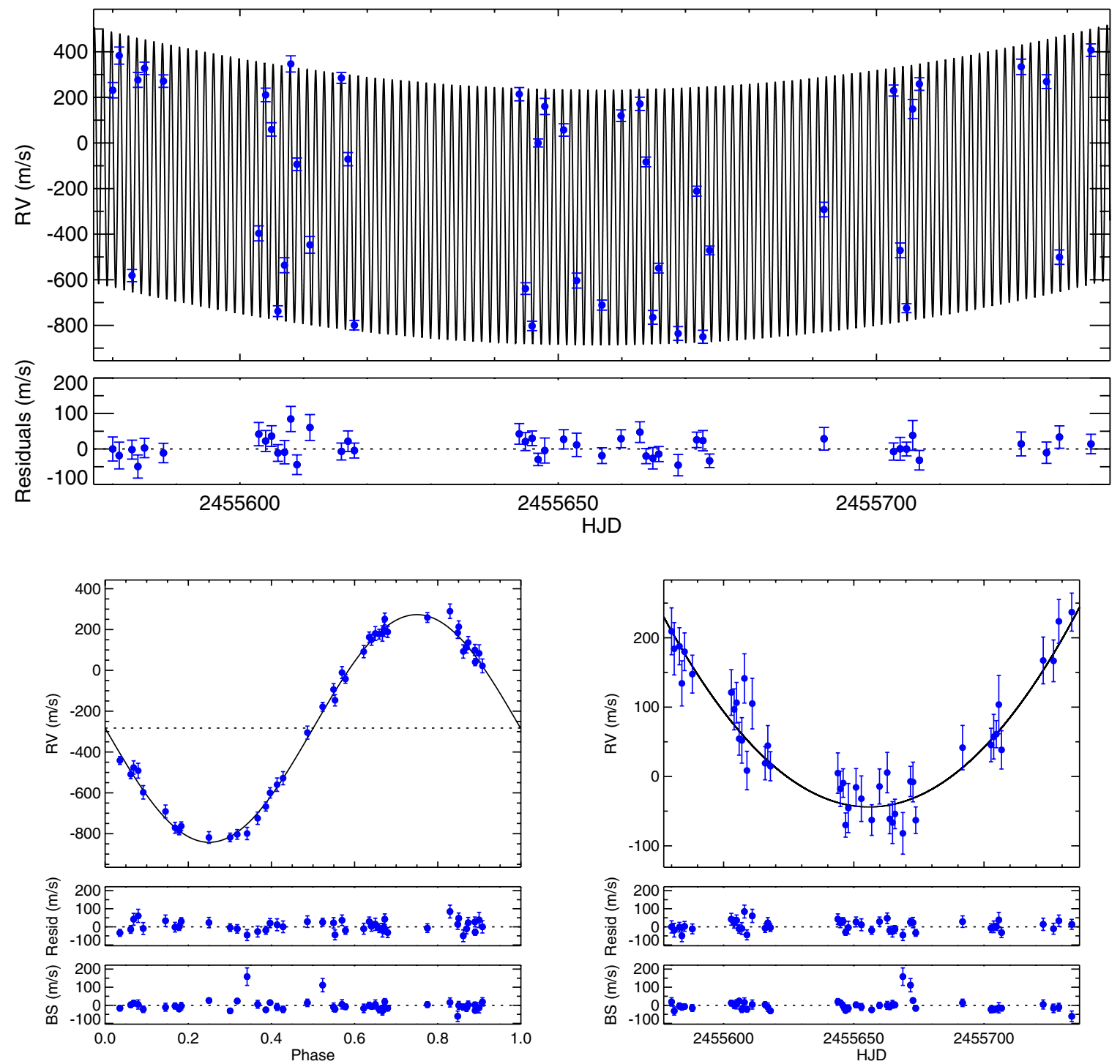

Figure 2. Velocity curve for the orbit of Qatar- $2 b$ (on the bottom left, phased to the period from the orbital fit) and for the quadratic residuals due to Qatar-2c (on the bottom right), together with the corresponding observed velocities. The top panel shows the original velocities together with the combined velocity model. The panels immediately under the velocity curves show the residuals from the fits. The variations in the bisector spans are plotted in the very bottom panels.

(A color version of this figure is available in the online journal.)

longitude of periastron or the time of periastron in Table 2. Note that the period of the inner orbit from the radial velocities alone, $P_{\mathrm{RV}}=1.337169 \pm 0.000076$, is a close match to the period from the final global solution together with all the photometry, $P=1.3371182 \pm 0.0000037$ days. Furthermore, the solution for the outer companion is suggestive, but it is premature to quantify how reliable it is. The Systemic Console yielded several plausible solutions, whose differences were much greater than the internal error estimates. A reliable solution of the orbit of Qatar-2c will require additional observations, which are planned for the coming season.

\subsection{Bisector Analysis}

The radial velocity of a star is defined to be the velocity of the center of mass of the star along the line of sight. Observationally, radial velocities are determined by measuring the Doppler shifts of spectral lines formed in the star's atmosphere. Distortions of line profiles due to phenomena such as dark spots on the surface of a rotating star or contamination of the spectrum by another star with variable velocity can mimic radial-velocity variations in the target star. A common technique for detecting line profile variations involves measurements of line bisector spans (e.g., see Queloz et al. 2001; Torres et al. 2007). Any variation in the bisector spans that is correlated with variations in the radial velocities is a strong warning that the velocity variation is probably not due to the reflex motion induced by an orbiting planet.

The variations in the bisector spans that we measured for the 44 TRES observations of Qatar-2 are reported in Table 1 and are plotted in the bottom panels of Figure 2. We were particularly interested to see if the bisector spans showed a correlation with the quadratic drift attributed to Qatar-2c, as might be expected if the outer companion contributed significant light. That would be compelling evidence that Qatar-2c was actually a star. No such trend is apparent in the bisector spans. There is also no correlation with the period of Qatar-2b, supporting the planet interpretation. However, there are two outliers in the bisector spans which cannot be readily explained. This is another motivating factor for getting more observations, to determine if the bisectors of those are well behaved. 


\subsection{Stellar Parameter Classification (SPC)}

The TRES spectra were also used to classify the stellar parameters of Qatar-2 using Stellar Parameter Classification (SPC), a new tool (L. A. Buchhave et al., in preparation) for comparing an observed spectrum with a library of synthetic spectra. SPC has its origins in the procedures developed for the analysis of spectra obtained with the CfA Digital Speedometers (cf. Carney et al. 1987; Latham et al. 2002). It is designed to solve simultaneously for effective temperature $\left(T_{\text {eff }}\right)$, metallicity $([\mathrm{m} / \mathrm{H}])$, surface gravity $(\log g)$, projected rotational velocity $(v \sin i$ ), and radial velocity (RV), taking advantage of the higher quality and more extensive wavelength coverage of the spectra produced by modern CCD echelle spectrographs. In essence, SPC cross-correlates an observed spectrum with a library of synthetic spectra for a grid of Kurucz model atmospheres and finds the stellar parameters by determining the extreme of a multi-dimensional surface fit to the peak correlation values from the grid.

For the SPC analysis of Qatar-2, we shifted the 44 observed spectra to a common velocity and co-added them, to obtain a combined observed spectrum with SNRe of 175 in the continuum near the $\mathrm{Mg}$ b features at $518 \mathrm{~nm}$. An SPC analysis yielded $T_{\text {eff }}=4610 \pm 50 \mathrm{~K}, \log g=4.65 \pm 0.10(\mathrm{cgs})$, $[\mathrm{m} / \mathrm{H}]=-0.02 \pm 0.08$, and $v \sin i=2.8 \pm 0.5 \mathrm{~km} \mathrm{~s}^{-1}$. The errors quoted here are our best guesses at the limit set by the systematic errors, which dominate the internal errors estimated from the consistency of the results for the individual observations. These values of $T_{\text {eff }}$ and $[\mathrm{m} / \mathrm{H}]$ are inputs to the determination of the mass and radius of the host star (not to mention its age) using stellar models, as described in Section 6. This is an important issue because the accuracy of the mass and radius determined for a transiting planet is often limited by uncertainties in the mass and radius of its host star.

\section{FOLLOW-UP PHOTOMETRY}

\subsection{KeplerCam Observations}

Light curves for four transits of Qatar-2b were obtained by use of KeplerCam on the $1.2 \mathrm{~m}$ telescope at the Fred Lawrence Whipple Observatory on Mount Hopkins, Arizona. KeplerCam utilizes a single Fairchild $4864 \mathrm{~K} \times 4 \mathrm{~K}$ CCD to cover an area of $23^{\prime} \times 23^{\prime}$ on the sky, with a typical FWHM of $2^{\prime \prime} .5$ for stellar images. We observed transit events of Qatar- $2 b$ on the nights of 2011 February 26, 2011 March 6, 2011 March 14, and 2011 March 18. The number of images captured for each of these events was 79, 109, 94, and 168, respectively. A Sloan Digital Sky Survey (SDSS) $i$-band filter was used for three of the transits, and an SDSS $g$-band filter was used for the fourth. For one of the $i$-band light curves we only covered half a transit, due to increasing cloud cover around the time of egress. The $g$-band light curve was acquired in order to look for possible color effects due to light from additional stars contaminating the Qatar-2 image, such as a background eclipsing binary.

The goal of the KeplerCam observations was to produce highquality light curves, model them, and derive values for the radius ratio of the planet to the star, $R_{\mathrm{P}} / R_{\star}$; the scaled semimajor axis of the orbit, $a / R_{\star}$; and orbital inclination, $i$ (or equivalently the impact parameter, $b$ ). Differential aperture photometry was carried out on the images, after the usual steps of bias subtraction and flat fielding. We experimented with the sizes used for the aperture for the stellar images and the annulus used for the sky subtraction. A smaller aperture reduces the contribution of sky background to the noise, but risks larger systematic errors
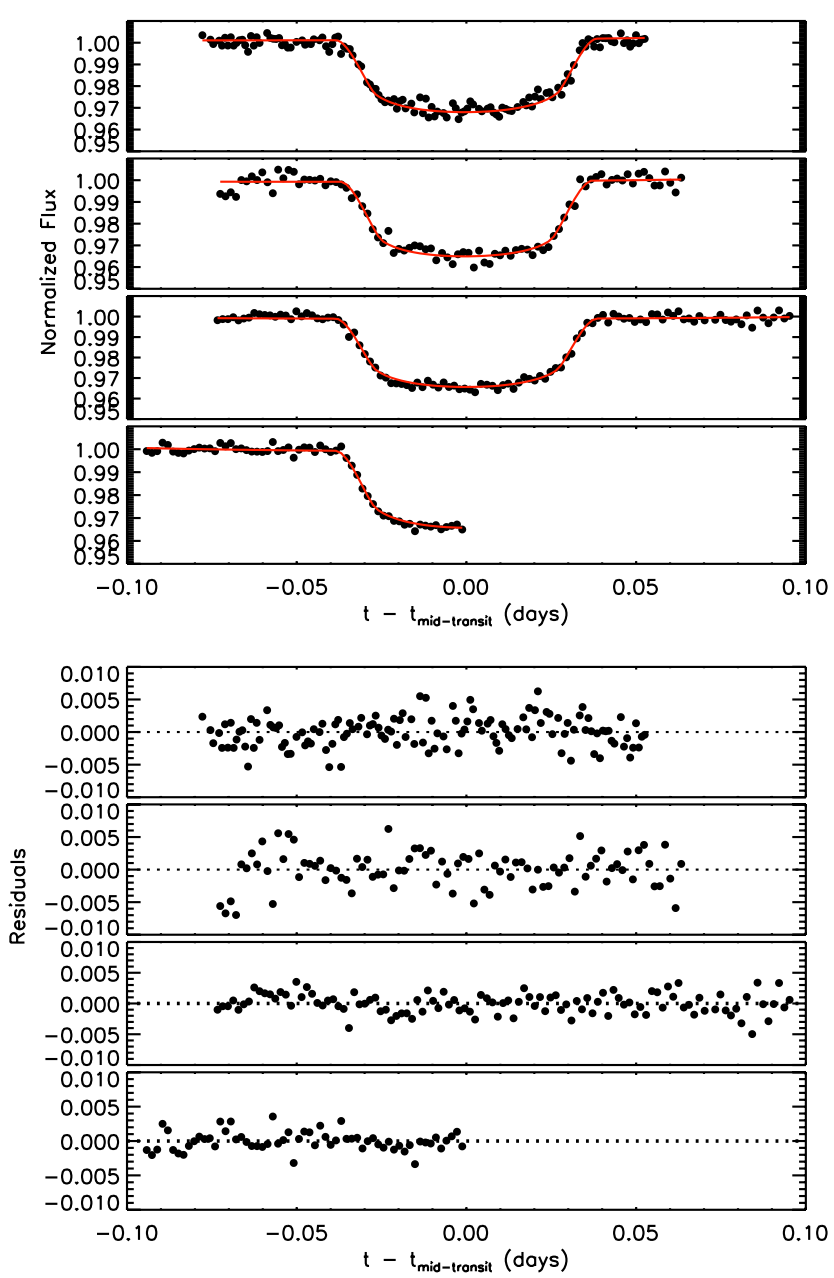

Figure 3. KeplerCam light curves for the transits of Qatar-2b. The second light curve from the top was obtained with an SDSS $g$ filter, the other three were obtained with an SDSS $i$ filter. The residuals from the model are shown underneath the light curves. The exposure times were $120 \mathrm{~s}$ for the $g$ band and 60 for the $i$ band.

(A color version of this figure is available in the online journal.)

due to imperfect centering of the stellar image. We chose the aperture and annulus sizes that gave the best balance between these two competing sources of error; $8^{\prime \prime}-9^{\prime \prime}$ in diameter for the stellar aperture, and an annulus between $30^{\prime \prime}$ and $60^{\prime \prime}$ for the sky. Sections of the out-of-transit light curves that showed contamination by incoming clouds or dawn were rejected. Nine reference stars were identified, and the sum of their light curves was divided into the light curve for Qatar-2 in order to correct for atmospheric and instrumental effects. A linear trend was then fit to the out-of-transit sections of the resulting differential light curve and was used to normalize the light curve for Qatar-2b to unity.

Figure 3 shows the KeplerCam light curves together with the model fits described in Section 6. The residuals of the photometric data from the model light curves are plotted below the light curves and show very little correlated noise. In addition, plots of the data against column and row position on the CCD showed no obvious correlation, and thus are not reproduced here.

\subsection{LCOGT Observations}

Four partial transit events were observed with a $0.8 \mathrm{~m}$ telescope operated by the Las Cumbres Observatory Global 
Telescope (LCOGT) at the Byrne Observatory at Sedgwick Reserve near Santa Ynez, CA. The Sedgwick telescope is equipped with a Santa Barbara Instrument Group STL-6303E camera utilizing a $3 \mathrm{~K} \times 2 \mathrm{~K}$ Kodak Enhanced KAF-6306E CCD with a pixel scale of $0^{\prime \prime} .572$ pixel $^{-1}(2 \times 2$ binning $)$, and a $14^{\prime} .7 \times 9^{\prime} .8$ field of view. We observed in the SDSS $r$ band with exposure times of $120 \mathrm{~s}$.

The images were reduced using standard routines for bias subtraction, dark current subtraction, and flat-field correction. We extracted instrumental fluxes for the stellar images using PyRAF and aperture photometry. Relative light curves were produced by dividing the fluxes of the target star by the sum of the fluxes of four comparison stars in each image. Each transit event was normalized to the out-of-transit flux on that particular night. Julian dates of mid-exposure were recorded during the observations, and later converted to BJD TDB using the online versions of the tools described by Eastman et al. (2010). Aperture sizes, between $4^{\prime \prime}$ and $7^{\prime \prime}$ depending on the image quality, were chosen in order to minimize the photometric residuals in the resulting light curves.

\section{HIGH-RESOLUTION IMAGES}

A common source of astrophysical false positives for planet candidates discovered by wide-angle photometric surveys is contamination of the target image by an eclipsing binary, either by a physical companion in a hierarchical system or by an accidental alignment with a background binary. Groundbased surveys that utilize cameras with short focal lengths are particularly vulnerable to this problem because the detector pixels typically span $10^{\prime \prime}$ or more on the sky. The $400 \mathrm{~mm}$ focal length Canon lenses used by the QES cameras produce images with a typical FWHM of 7".5. This is roughly half the size of the images produced by the $200 \mathrm{~mm}$ Canon lenses used by several ground-based surveys, and thus the rate of contamination by background eclipsing binaries should be four times better. The images of the KeplerCam follow-up photometry have a typical FWHM of 2.5 , so this should provide nearly an orderof-magnitude additional improvement in the contamination rate.

To push to even lower limits, we obtained high-resolution images with the Danish $1.54 \mathrm{~m}$ telescope at the European Southern Observatory on La Silla, by use of an Andor iXon ${ }^{\mathrm{EM}}+897$ camera with an EMCCD chip, often called a Lucky-Imagingcamera because of its ability to obtain diffraction-limited images by recording lots of short exposures and collecting together the best images. The camera has a pixel size corresponding to 0.09 , and was read at a frame rate of $10 \mathrm{~Hz}$. In order to reach diffraction-limited images, one would usually stack the few percentage best quality images from a sequence, but here we have just applied the shift and add technique to all the images in a sequence of 1,000 individual exposures, which typically will reduce the seeing by a factor of about three compared to traditional CCD observations with the same total exposure time.

Figure 4 shows two of the resulting images. Qatar- 2 is the bright star in the upper right part (i.e., northwest) of the images. The two fainter stars in the lower part of the image approximately $36^{\prime \prime}$ southwest of Qatar-2 are separated by slightly less than 0.'6. The FWHM of the image of Qatar-2 is about 0.'5, so these images reduce the area on the sky that could be contaminated by a background eclipsing binary by nearly another order of magnitude compared to the KeplerCam images. Although these images reduce the chance of a false positive due to a background eclipsing binary to a negligible level, they do not reduce significantly the chance of a false positive due to contamination

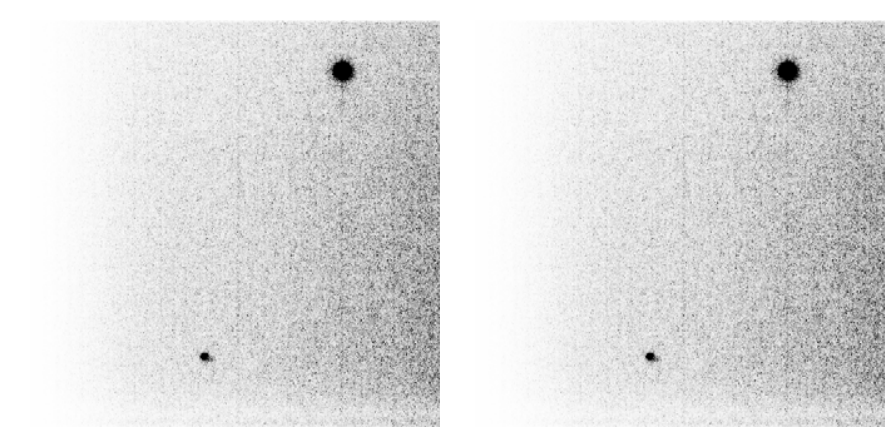

Figure 4. Lucky camera images of Qatar-2. North is up and west is left. The faint pair of stars $36^{\prime \prime}$ to the southwest are separated by slightly less than 0 .' 6 .

in a hierarchical system because the angular separation of most such systems is below the resolution of these images.

\section{PHYSICAL CHARACTERISTICS OF THE STAR AND INNER PLANET}

The stellar mass and radius for Qatar-2 were initially estimated using the values of $T_{\text {eff }}$ and $[\mathrm{m} / \mathrm{H}]$ derived from the spectra using SPC and the value for $a / R_{\star}$ derived from the KeplerCam light curves, together with isochrones from the Yonsei-Yale series of stellar models (Yi et al. 2001), following the procedures described by Torres et al. (2008). This yielded a mass of $M_{\star}=0.742 \pm 0.35 M_{\odot}$, which was then imposed as an MCMC parameter controlled by a Gaussian prior on the simultaneous fit to the radial velocities and all the light curves from the QES, KeplerCam, and LCOGT photometry. All of our exploratory fits to the radial velocities indicated that the orbital eccentricity for Qatar-2b was indistinguishable from circular, so in our final model we fixed that eccentricity to zero and fit the residuals of the radial velocities from a circular orbit using a quadratic Taylor expansion. The transit light curves were modeled using the formulation of Mandel \& Agol (2002) in the small-planet approximation. A four-coefficient nonlinear limb-darkening model was used, employing fixed coefficients appropriate to the $R$ band for the QES photometry, the SDSS $r$ band for the LCOGT photometry, and the SDSS $i$ or $g$ band for the KeplerCam photometry. These coefficients were determined from the tables of Claret (2004), interpolated to the values of $T_{\text {eff }}$, and $[\mathrm{m} / \mathrm{H}]$ determined from the TRES spectra using SPC.

The parameter optimization was performed using the current version of the MCMC code described by Collier Cameron et al. (2007) and Pollacco et al. (2008). The transit light curve is modeled in terms of the epoch $T_{0}$ of mid transit, the orbital period $P$, the ratio of the radii squared $d=\left(R_{\mathrm{P}} / R_{\star}\right)^{2}$, the approximate duration $t_{T}$ of the transit from initial to final contact, and the impact parameter $b=(a \cos i) / R_{\star}$. The radial-velocity model is defined by the stellar orbital velocity semi-amplitude due to the inner planet $K_{b}$ and three coefficients in a quadratic Taylor expansion for the acceleration of the star due to the outer planet, the first coefficient being the offset of the centerof-mass velocity for the relative velocities used for the orbital solution, $\Delta \gamma$. The values of $T_{\text {eff }}$ and $[\mathrm{m} / \mathrm{H}]$ were treated as additional MCMC parameters, constrained by Gaussian priors with mean values and variances as determined from the TRES spectra using SPC. The final light curves and corresponding fits from the global analysis are shown in Figure 5 for the QES discovery photometry and for the KeplerCam and LCOGT follow-up photometry. The correlations between the various posterior parameter estimates are shown in the matrix of plots 

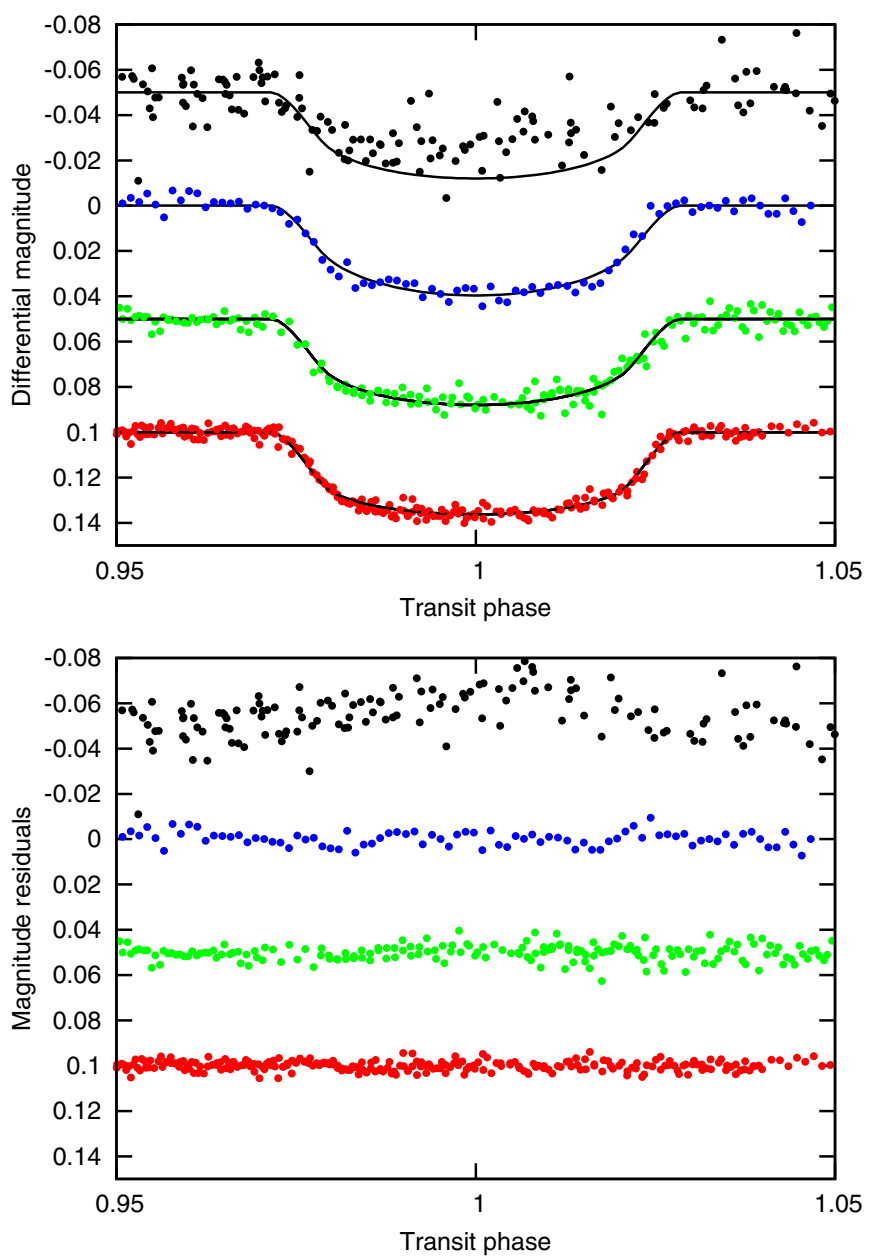

Figure 5. Fit of the light curves found by the global analysis. The first curve in the upper panel shows the QES discovery light curve in the $R$ band; the second shows the KeplerCam SDSS $g$-band light curve; the third shows the LCOGT SDSS $r$-band light curve, and the fourth shows the KeplerCam SDSS $i$-band light curve. The lower panel shows the corresponding residuals from the global fit. The dip in the QES light curve may be too shallow due to aggressive detrending. The final photometric data for the KeplerCam, QES, and LCOGT light curves are provided in full in the online version of the journal.

(A color version and FITS images of this figure are available in the online journal.)

in Figure 6. The final physical and orbital parameters and error estimates for the star and planet are reported in Table 3. It is reassuring to see the good agreement between the stellar parameters estimated from the spectra using SPC and the final values from the global analysis: $T_{\text {eff }}=4650 \pm 50 \mathrm{~K}$ from SPC compared to $4645 \pm 50$, and $\log g=4.65 \pm 0.10 \mathrm{cgs}$ versus $4.601 \pm 0.018$.

\section{DISCUSSION}

\subsection{Hot Jupiters with Companions}

One of the most important results from the Kepler mission is the discovery of a rich population of multiple transiting planets in systems whose orbits must be coplanar within a degree or two (Latham et al. 2011; Lissauer et al. 2011). Especially striking is the very low occurrence rate for hot Jupiters in the multiple systems found by Kepler. In the sample of 1235 planet candidates announced by Borucki et al. (2011), there are 117 systems that contain a planet candidate more massive than Saturn $\left(M_{\mathrm{P}}>0.3 M_{\mathrm{J}}\right)$ in an orbit with period shorter than 10 days, but only 5 of these systems harbor an additional transiting planet candidate. The remaining 112 transiting hot Jupiters are all in singles.

The occurrence rate is also very low for companions to hot Jupiters found by ground-based surveys, both photometric and spectroscopic. A review of the Extrasolar Planet Encyclopaedia (Schneider 2011) reveals that Qatar-2b joins HAT-P-13b, HAT-P-17b, and HAT-P-31b as the fourth transiting hot Jupiter with a confirmed outer planet, while only four hot Jupiters found by radial velocities have outer companions (HD 217107b, HIP $14810 \mathrm{~b}, \mathrm{HD} 187123 \mathrm{~b}$, and $v$ Andb). Of course, additional radialvelocity monitoring may reveal other hot Jupiters with outer companions, and there are already hints of velocity drifts for WASP-8 and WASP-22. All of the confirmed outer companions have relatively long orbital periods (see Table 4), so it is not surprising that none of them have shown transits, so far. Nevertheless, it is tempting to speculate that a close-in giant planet may stir up the orbits of other inner planets in its system, while a system of small planets is more likely to preserve the flatness of the disk from which it formed, allowing small planets to survive in surprisingly compact configurations.

In only one of the systems (HIP 14810) listed in Table 4 is the outer companion less massive than the hot Jupiter. In the rest of the systems, the nearest companions are more massive, often by large factors.

\subsection{Orbits of Qatar- $2 b$ and HAT-P-31b}

Why is the orbit of HAT-P-31b eccentric, with $e=0.2450 \pm$ 0.0045 (Kipping et al. 2011), while the orbit of Qatar-2b is indistinguishable from circular? One possible explanation is that both orbits started out with significant eccentricity, perhaps as the result of a dynamical encounter that sent each planet on a path close to its host star, and the orbit of Qatar-2b has since been circularized by tidal forces, while the orbit of HAT-P-31b has not.

The circularization timescale goes something like $\left(M_{\mathrm{P}} / M_{\star}\right)$ $\left(a / R_{\star}\right)^{5}$. HAT-P-31b has a period of $P=5.00$ days and mass of $M_{\mathrm{P}}=2.17 M_{\mathrm{J}}$, orbits a late F star with mass $M_{\star}=1.22 M_{\odot}$, $a / R_{\star}=8.9$, and is about $3 \mathrm{Gyr}$ old. In contrast, Qatar-2b has a much shorter period of $P_{\mathrm{b}}=1.34$ days, similar mass of $M_{\mathrm{P}}=2.49 M_{\mathrm{J}}$, orbits a $\mathrm{K}$ dwarf with mass of $M_{\star}=0.74 M_{\odot}$, $a / R_{\star}=6.3$, and is very likely a member of the Galactic disk. If Qatar-2 is three times older than HAT-P-31, the system age divided by the circularization timescale is nearly an order of magnitude longer for Qatar-2b, long enough so that there has been time for tidal forces to circularize the orbit. In addition, the host star for HAT-P-31b is close to the mass where the outer envelope no longer supports a convection zone, and the circularization timescale is even longer than implied by the simple relation that we adopted for the sake of this discussion.

Another effect that needs to be explored more carefully with dynamical simulations is the amount of eccentricity that Qatar2c can pump into the orbit of Qatar-2b. Are the tidal forces working to circularize the orbit of Qatar-2b strong enough to keep the orbit circularized despite the perturbations from Qatar$2 \mathrm{c}$ ? Finally, it would be interesting to estimate the size and patterns of the transit time variations expected for Qatar-2b due to perturbations by Qatar-2c.

\subsection{The Value of Deep Transits}

With a depth of about 3.5\%, the transits of Qatar-2b are deeper than the transits of any other planet listed in 


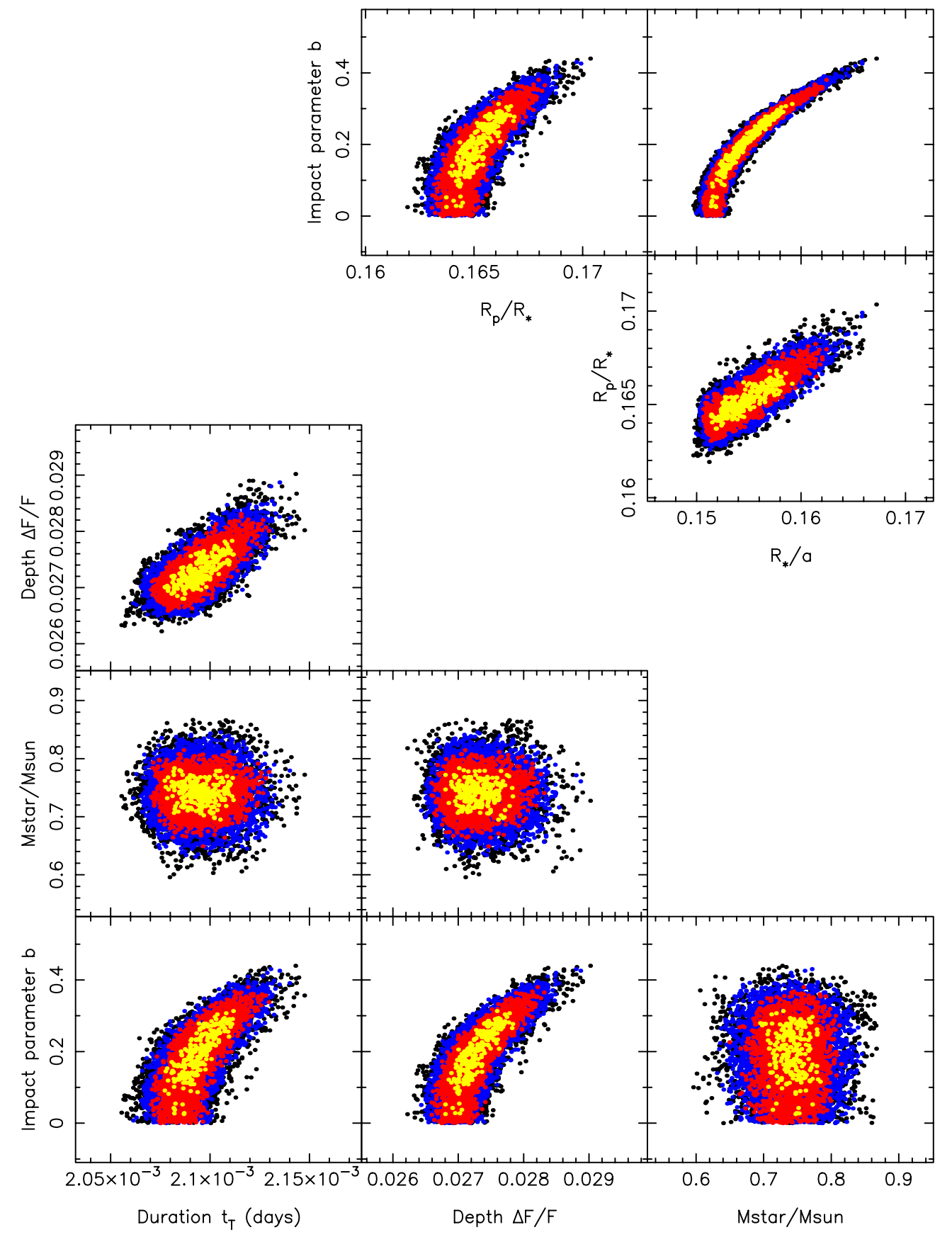

Figure 6. Correlations between the various posterior estimates from the MCMC global fit.

(A color version of this figure is available in the online journal.)

The Extrasolar Planets Encyclopaedia (Schneider 2011). The closest rival is CoRoT-2b, with reported transit depths of $3.2 \%$ (Alonso et al. 2008). CoRoT-2 is a late $\mathrm{G}$ dwarf with $T_{\text {eff }}=$ $5625 \mathrm{~K}$, while Qatar- 2 is a late $\mathrm{K}$ dwarf with $T_{\text {eff }}=4645 \mathrm{~K}$; the smaller radius of Qatar-2 is the main reason for the deeper transits. A more interesting comparison may be the bright ( $V=7.7 \mathrm{mag}$ ) early K dwarf HD 189733 with $T_{\text {eff }}=5050 \mathrm{~K}$ (cf. Bouchy et al. 2005; Bakos et al. 2006), which has been a favorite target for studies of the planet's atmosphere (cf. Knutson et al. 2007; Swain et al. 2008; Grillmair et al. 2008; Gibson et al. 2011). The contrast between the planet and host star is even more favorable for Qatar-2, but the system is much fainter $(V=13.3 \mathrm{mag})$. Thus, HD 189733 is likely to continue as a top target for follow-up studies of hot Jupiters, although it may eventually be joined by other nearby bright transiting systems discovered by all-sky transit surveys such as TESS or PLATO, or by targeted searches of small cool stars such as MEarth. Nevertheless, Qatar-2 is a good target for amateurs and outreach projects such as MicroObservatory (http://mo-www.harvard.edu/MicroObservatory/) with telescopes of modest size but covering wide fields of view because of the availability of many more reference stars of comparable magnitude.

G.F. acknowledges financial support from the Hungarian OTKA-NFU Mobility grant MB08C 81013. C.L. acknowledges the Qatar Foundation for support from 
Table 3

Planetary and Stellar Parameters

\begin{tabular}{|c|c|c|c|}
\hline Parameter & Symbol & Value & Units \\
\hline Transit epoch (BJD TDB) & $T_{0}$ & $2455624.26679 \pm 0.00011$ & days \\
\hline Orbital period & $P_{\mathrm{b}}$ & $1.3371182 \pm 0.0000037$ & days \\
\hline Transit duration & $t_{T}$ & $0.07540 \pm 0.00049$ & days \\
\hline Planet/star area ratio & $\left(R_{\mathrm{P}} / R_{\star}\right)^{2}$ & $0.02725 \pm 0.00040$ & \\
\hline Impact parameter & $b$ & $0.19 \pm 0.10$ & $R_{*}$ \\
\hline Scaled stellar radius & $R_{\star} / a$ & $0.1541 \pm 0.0030$ & \\
\hline Stellar density & $\rho_{\star}$ & $2.05 \pm 0.12$ & $\rho_{\odot}$ \\
\hline Stellar effective temperature & $T_{\text {eff }}$ & $4645 \pm 50$ & $\mathrm{~K}$ \\
\hline Spectroscopic Metallicity & {$[\mathrm{m} / \mathrm{H}]$} & $-0.02 \pm 0.08$ & \\
\hline Stellar surface gravity & $\log g_{\star}$ & $4.601 \pm 0.018$ & (cgs) \\
\hline Projected stellar rotation speed & $v \sin i$ & 2.8 (fixed) & $\mathrm{km} \mathrm{s}^{-1}$ \\
\hline Stellar radius & $R_{\star}$ & $0.713 \pm 0.018$ & $R_{\odot}$ \\
\hline Stellar mass & $M_{\star}$ & $0.740 \pm 0.037$ & $M_{\odot}$ \\
\hline Orbital separation & $a$ & $0.02149 \pm 0.00036$ & $\mathrm{AU}$ \\
\hline Orbital inclination & $i$ & $88.30 \pm 0.94$ & $\circ$ \\
\hline Stellar reflex velocity & $K_{\mathrm{b}}$ & $558.7 \pm 5.9$ & $\mathrm{~ms}^{-1}$ \\
\hline Center-of-mass velocity offset & $\Delta \gamma$ & $-282.6 \pm 4.0$ & $\mathrm{~ms}^{-1}$ \\
\hline Drift in center-of-mass velocity & $d \gamma / d t$ & $-2.74 \pm 0.15$ & $\mathrm{~ms}^{-1} \mathrm{~d}^{-1}$ \\
\hline Quadratic velocity drift term & $d^{2} \gamma / d t^{2}$ & $0.0870 \pm 0.0043$ & $\mathrm{~ms}^{-1} \mathrm{~d}^{-2}$ \\
\hline Orbital eccentricity & $e$ & 0 (fixed) & \\
\hline Planet radius & $R_{\mathrm{p}}$ & $1.144 \pm 0.035$ & $R_{\mathrm{J}}$ \\
\hline Planet mass & $M_{\mathrm{p}}$ & $2.487 \pm 0.086$ & $M_{\mathrm{J}}$ \\
\hline Planet surface gravity & $\log g_{\mathrm{p}}$ & $3.638 \pm 0.022$ & (cgs) \\
\hline Planet density & $\rho_{\mathrm{p}}$ & $1.66 \pm 0.13$ & $\rho_{\mathrm{J}}$ \\
\hline Planetary equilibrium temperature & $T_{\mathrm{P}}$ & $1292 \pm 19$ & $\mathrm{~K}$ \\
\hline
\end{tabular}

Table 4

Hot Jupiters with Companions

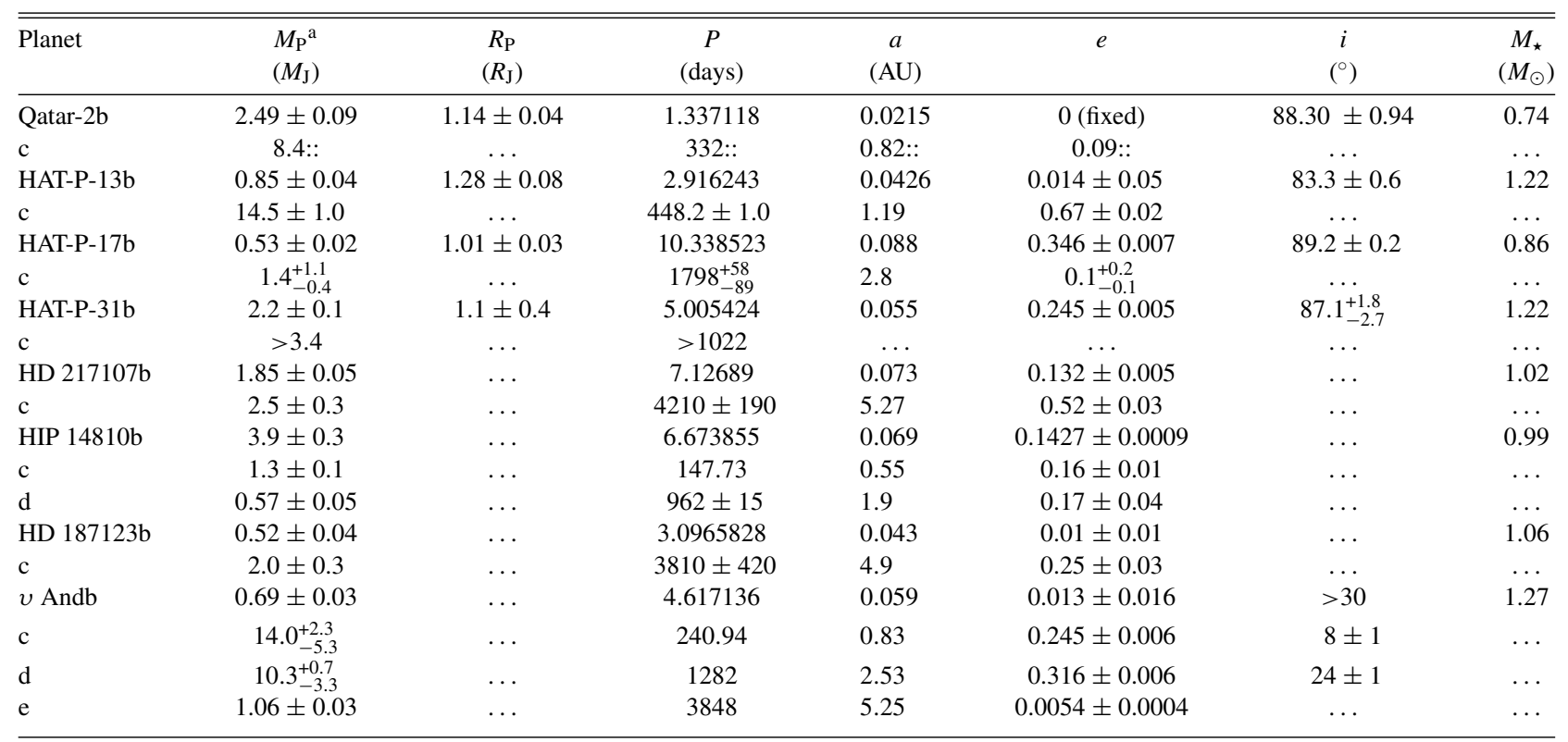

Note. ${ }^{\text {a }}$ For the transiting hot Jupiters (the first four entries), the actual mass is reported; for the hot Jupiters discovered by Doppler surveys (the final four entries), the minimum mass is listed, except for $v$ And $c$ and d, which are actual masses enabled by inclinations determined with the Fine Guidance Sensors on HST.

QNRF grant NPRP-09-476-1-078. D.R. (boursier FRIA) acknowledges support from the Communauté française de Belgique-Actions de recherche concertées-Académie universitaire Wallonie-Europe. The Byrne Observatory at Sedgwick (BOS) is operated by the Las Cumbres Observatory Global Telescope Network and is located at the Sedgwick Reserve, a part of the University of California Natural Reserve System. We thank the anonymous referee for several helpful suggestions for improving the clarity of the manuscript and the interpretation of the results.

$$
\text { Facility: FLWO:1.5m (TRES) }
$$

\section{REFERENCES}

Alonso, R., Auvergne, M., Baglin, A., et al. 2008, A\&A, 482, L21 Alsubai, K. A., Parley, N. R., Bramich, D. M., et al. 2011, MNRAS, 417, 709 
Bakos, G. Á., Knutson, H., Pont, F., et al. 2006, ApJ, 650, 1160

Batalha, N. M., Borucki, W. J., Bryson, S. T., et al. 2011, ApJ, 729, 27

Bean, J. L., Désert, J.-M., Kabath, P., et al. 2011, ApJ, 743, 92

Borucki, W. J., Koch, D. G., Basri, G., et al. 2011, ApJ, 736, 19

Bouchy, F., Udry, S., Mayor, M., et al. 2005, A\&A, 444, L15

Bramich, D. M. 2008, MNRAS, 386, L77

Buchhave, L. A., Bakos, G. Á., Hartman, J. D., et al. 2010, ApJ, 720, 1118

Carney, B. W., Laird, J. B., Latham, D. W., \& Kurucz, R. L. 1987, AJ, 93, 116

Charbonneau, D., Berta, Z. K., Irwin, J., et al. 2009, Nature, 462, 891

Claret, A. 2004, A\&A, 428, 1001

Collier Cameron, A., Pollacco, D., Street, R. A., et al. 2006, MNRAS, 373, 799

Collier Cameron, A., Wilson, D. M., West, R. G., et al. 2007, MNRAS, 380, 1230

Eastman, J., Siverd, R., \& Gaudi, B. S. 2010, PASP, 122, 935

Gibson, N., Pont., F., \& Aigrain, S. 2011, MNRAS, 411, 2186

Grillmair, C., Burrows, A., Charbonneau, D., et al. 2008, Nature, 456, 767

Kipping, D. M., Hartman, J., Bakos, G. Á., et al. 2011, AJ, 142, 95

Knutson, H., Charbonneau, D., Allen, L. E., et al. 2007, Nature, 447, 183
Kovács, G., Bakos, G. Á., \& Noyes, R. W. 2005, MNRAS, 356, 557

Kovács, G., Zucker, S., \& Mazeh, T. 2002, A\&A, 391, 369

Latham, D. W., Rowe, J. F., Quinn, S. N., et al. 2011, ApJ, 732, L24

Latham, D. W., Stefanik, R. P., Torres, G., et al. 2002, AJ, 124, 1144

Lissauer, J. J., Ragozzine, D., Fabrycky, D. C., et al. 2011, ApJS, 197, 8

Mandel, K., \& Agol, E. 2002, ApJ, 580, 171

Meschiari, S., Wolf, A. S., Rivera, E., et al. 2009, PASP, 121, 1016

Pollacco, D. L., Skillen, I., Collier Cameron, A., et al. 2006, PASP, 118, 1407

Pollacco, D. L., Skillen, I., Collier Cameron, A., et al. 2008, MNRAS, 385, 1576

Queloz, D., Henry, G. W., Sivan, J. P., et al. 2001, A\&A, 379, 279

Schneider, J. 2011, http://exoplanet.eu (as of 2011 September 24)

Swain, M., Vasisht, G., \& Tinetti, G. 2008, Nature, 463, 637

Tamuz, O., Mazeh, T., \& Zucker, S. 2005, MNRAS, 356, 1466

Torres, G., Bakos, G. Á., Kovács, G., et al. 2007, ApJ, 666, 121

Torres, G., Winn, J. N., \& Holman, M. J. 2008, ApJ, 677, 1324

Yi, S. K., Demarque, P., Kim, Y.-C., et al. 2001, ApJS, 136, 417

Zacharias, N., Finch, C., Girard, T., et al. 2010, AJ, 139, 2184 


\title{
ERRATUM: “QATAR-2: A K DWARF ORBITED BY A TRANSITING HOT JUPITER AND A MORE MASSIVE COMPANION IN AN OUTER ORBIT” (2012, ApJ, 750, 84)
}

\author{
Marta L. Bryan ${ }^{1}$, Khalid A. Alsubai ${ }^{2}$, David W. Latham ${ }^{3}$, Neil R. Parley ${ }^{4}$, Andrew Collier Cameron ${ }^{4}$, \\ Samuel N. Quinn ${ }^{3}$, Joshua A. Carter ${ }^{3}$, Benjamin J. Fulton ${ }^{5}$, Perry Berlind ${ }^{3}$, Warren R. Brown ${ }^{3}$, \\ Lars A. Buchhave ${ }^{6,7}$, Michael L. Calkins ${ }^{3}$, Gilbert A. Esquerdo ${ }^{3}$, Gábor Fúrész ${ }^{3,8}$, Uffe Gråe Jørgensen ${ }^{6,7}$, \\ Keith D. Horne ${ }^{4}$, Robert P. StefaniK ${ }^{3}$, Rachel A. Street ${ }^{5}$, Guillermo Torres ${ }^{3}$, Richard G. West ${ }^{9}$, Martin Dominik ${ }^{4,13}$, \\ Kennet B. W. Harpsøe ${ }^{6,7}$, Christine Liebig ${ }^{4}$, Sebastiano Calchi Novati ${ }^{10,11}$, Davide Ricci ${ }^{12}$, and Jesper F. Skottfelt ${ }^{6,7}$ \\ ${ }^{1}$ Department of Astronomy, Harvard University, Cambridge, MA 02138, USA \\ ${ }^{2}$ Qatar Foundation, P.O. Box 5825, Doha, Qatar \\ ${ }^{3}$ Harvard-Smithsonian Center for Astrophysics, 60 Garden Street, Cambridge, MA 02138, USA \\ ${ }^{4}$ SUPA, School of Physics and Astronomy, University of St Andrews, North Haugh, St Andrews, Fife KY16 9SS, UK \\ ${ }^{5}$ Las Cumbres Observatory Global Telescope Network, 6740 Cortona Drive, Suite 102, Goleta, CA 93117, USA \\ ${ }^{6}$ Niels Bohr Institute, University of Copenhagen, Juliane Maries vej 30, DK-2100 Copenhagen, Denmark \\ ${ }^{7}$ Centre for Star and Planet Formation, Geological Museum, Øster Voldgade 5, DK-1350 Copenhagen, Denmark \\ ${ }^{8}$ Konkoly Observatory of the Hungarian Academy of Sciences, Budapest, Hungary \\ ${ }^{9}$ Department of Physics and Astronomy, University of Leicester, Leicester LE1 7RH, UK \\ ${ }^{10}$ Dipartimento di Fisica “E.R. Caianiello," Universita degli Studi di Salerno, Via S. Allende, I-84081 Baronissi (SA), Italy \\ ${ }^{11}$ INFN, Gruppo Collegato di Salerno, Sezione di Napoli, Italy \\ 12 Institut d'Astrophysique et de Géophysique, Allée du 6 Août 17, Sart Tilman, Bât. B5c, B-4000 Liège, Belgium \\ Received 2013 December 11; published 2014 February 4
}

When the discovery paper was published, residuals from the 1.34 day orbital solution indicated a companion with several possible periods. With additional radial velocity measurements over the following months, the authors became concerned that the period of the outer planet was converging on one year. After checking for potential sources of error, the authors determined that there was an error in the barycentric correction, and that the outer planet was a detection of Earth's orbital motion. Qatar- 2 was the first target observed by TRES with negative declination, and this uncovered a bug in the code that parsed the sexigesimal declination-the minutes and seconds of arc were parsed as positive instead of negative. This resulted in a low amplitude modulation of the barycentric correction due to Earth's motion around the Sun. No other published result from TRES is affected by this error. A reanalysis using the correct barycentric velocity shows that the orbital solution for Qatar-2b is unchanged from the results published in the discovery paper.

\footnotetext{
${ }^{13}$ Royal Society University Research Fellow.
} 